Research Article

\title{
Commutative $C^{*}$-Algebras of Toeplitz Operators via the Moment Map on the Polydisk
}

\author{
Mauricio Hernández-Marroquin, Armando Sánchez-Nungaray, and \\ Luis Alfredo Dupont-García
}

Facultad de Matemáticas, Universidad Veracruzana, Lomas del Estadio S/N, 91000 Xalapa, VER, Mexico

Correspondence should be addressed to Armando Sánchez-Nungaray; armsanchez@uv.mx

Received 1 March 2016; Accepted 24 April 2016

Academic Editor: Kehe Zhu

Copyright (c) 2016 Mauricio Hernández-Marroquin et al. This is an open access article distributed under the Creative Commons Attribution License, which permits unrestricted use, distribution, and reproduction in any medium, provided the original work is properly cited.

\begin{abstract}
We found that in the polydisk $\mathbb{D}^{n}$ there exist $(n+1)(n+2) / 2$ different classes of commutative $C^{*}$-algebras generated by Toeplitz operators whose symbols are invariant under the action of maximal Abelian subgroups of biholomorphisms. On the other hand, using the moment map associated with each (not necessary maximal) Abelian subgroup of biholomorphism we introduced a family of symbols given by the moment map such that the $C^{*}$-algebra generated by Toeplitz operators with this kind of symbol is commutative. Thus we relate to each Abelian subgroup of biholomorphisms a commutative $C^{*}$-algebra of Toeplitz operators.
\end{abstract}

\section{Introduction}

In recent years, Vasilevski, Quiroga, and coauthors found a connection that the commutative $C^{*}$-algebras generated by Toeplitz operators acting on the weighted Bergman space were described and classified for the case of the unit disk and the unit ball in $\mathbb{C}^{n}$; see $[1,2]$ for further results and details. The classification result states that given a maximal Abelian subgroup $G$ of biholomorphisms of the unit ball or the unit disk (i.e., ball of dimension one), the $C^{*}$-algebra generated by Toeplitz operators whose symbols are invariant under the action of $G$ is commutative on each weighted Bergman space.

For the unit ball of dimension $n$ there are $n+2$ models of maximal Abelian subgroups $G$ of biholomorphisms; all others are conjugated with one of them via biholomorphisms of the unit ball.

We apply the above method to the polydisk $\mathbb{D}^{n}$ and we find that there exist $(n+1)(n+2) / 2$ different classes of commutative $C^{*}$-algebras generated by Toeplitz operators whose symbols are invariant under the action of maximal Abelian subgroups of biholomorphisms. Moreover, we show that in each case the Toeplitz operator $T_{a}$ is unitary equivalent to a multiplication operator $\gamma_{a, k} I$. The explicit form of $\gamma_{a, k}$ is given.
The main result of this paper is that we relate to each Abelian subgroup (not necessary maximal) of biholomorphisms a $C^{*}$-algebra generated by Toeplitz operators. We consider the symplectic manifold $\left(\mathbb{D}^{n}, \omega\right)$ where $\omega$ is the usual symplectic form; using the fact that the action of biholomorphisms is Hamiltonian we give the moment map $\mu_{H}$ associated with each Abelian subgroup $H$, where $\mu_{H}: \mathbb{D}^{n} \rightarrow \mathfrak{h}^{*}$. Then we introduce the family of symbols $\widetilde{S}_{H}$ which are given by functions of the form $a \circ \mu_{H}$, where $a$ is an arbitrary bounded function defined on $\mu_{H}\left(\mathbb{D}^{n}\right)$. Finally, we prove that the $C^{*}$-algebra generated by Toeplitz operators with symbols in the family $\widetilde{S}_{H}$ is commutative.

One important advantage of the point of view of the moment map is that if we consider the symbol set invariant under the action of a nonmaximal Abelian subgroup of biholomorphisms, then the $C^{*}$-algebra generated by Toeplitz operators with symbols in this set may not be commutative. In a sense, this symbol set is too big for our purposes, so this method no longer works for nonmaximal Abelian subgroups.

The method to find commutative $C^{*}$-algebras of Toeplitz operators using the moment map has not been used in other manifolds and we suppose that we can apply this technique to other symplectic manifolds. 


\section{Weighted Bergman Space and Bergman Projection on the Polydisk}

Let $n \in \mathbb{N}$ be fixed. Throughout this paper we use the notation $z=\left(z_{1}, \ldots, z_{n}\right)$ for a typical element $z \in \mathbb{C}^{n}$ and $z_{j}=x_{j}+i y_{j}$, $j=1, \ldots, n$. The unit polydisk is defined by

$$
\mathbb{D}^{n}=\left\{z \in \mathbb{C}^{n}:\left|z_{j}\right|<1, j=1, \ldots, n\right\} .
$$

Let $-1<\lambda<+\infty$; the normalized $\lambda$-weighted volume measure $d V_{\lambda}$ is given by

$$
\begin{aligned}
& d V_{\lambda}(z) \\
& =(\lambda+1)^{n}\left(\prod_{j=1}^{n}\left(1-\left|z_{j}\right|^{2}\right)^{\lambda}\right) d A\left(z_{1}\right) \cdots d A\left(z_{n}\right),
\end{aligned}
$$

where $z \in \mathbb{D}^{n}$ and $d A\left(z_{j}\right)=(1 / \pi) d x_{j} d y_{j}, j=1, \ldots, n$.

The weighted Bergman space $\mathscr{A}_{\lambda}^{2}\left(\mathbb{D}^{n}\right)$ is the space of all holomorphic functions which belongs to $L^{2}\left(\mathbb{D}^{n}, d V_{\lambda}\right)$. It is well known (see, e.g., [3]) that $\mathscr{A}_{\lambda}^{2}\left(\mathbb{D}^{n}\right)$ is a closed subspace of $L^{2}\left(\mathbb{D}^{n}, d V_{\lambda}\right)$. The (weighted) Bergman projection $B_{\mathbb{D}^{n}}^{\lambda}$ : $L^{2}\left(\mathbb{D}^{n}, d V_{\lambda}\right) \rightarrow \mathscr{A}_{\lambda}^{2}\left(\mathbb{D}^{n}\right)$ has the form

$$
\left(B_{\mathbb{D}^{n}}^{\lambda} f\right)(z)=\int_{\mathbb{D}^{n}} f(\zeta) \overline{K_{\mathbb{D}^{n}}^{\lambda}(z, \zeta)} d V_{\lambda}(\zeta),
$$

where the (weighted) Bergman kernel is given by

$$
K_{\mathbb{D}^{n}}^{\lambda}(z, \zeta)=\prod_{j=1}^{n} \frac{1}{\left(1-\overline{z_{j}} \zeta_{j}\right)^{\lambda+2}} .
$$

From now on we denote by $k=\left(k_{1}, k_{2}, k_{3}\right)$ a vector of nonnegative integer numbers such that $n=k_{1}+k_{2}+k_{3}$. Denote by $\Pi$ the upper half-plane; that is,

$$
\Pi=\{z \in \mathbb{C}: \operatorname{Im}(z)>0\} .
$$

Let $M_{k}$ denote the following domain in $\mathbb{C}^{n}$ :

$$
M_{k}=\mathbb{D} \times \cdots \times \mathbb{D} \times \Pi \times \cdots \times \Pi=\mathbb{D}^{k_{1}} \times \Pi^{k_{2}} \times \Pi^{k_{3}} .
$$

Consider the Hilbert space $L^{2}\left(M_{k}, d V_{\lambda}^{k}\right)$, where the normalized $\lambda$-weighted volume measure has the form

$$
\begin{aligned}
d V_{\lambda}^{k}(w)= & (\lambda+1)^{n} \prod_{j=1}^{k_{1}}\left(1-\left|w_{j}\right|^{2}\right)^{\lambda} \\
& \cdot \prod_{j=k_{1}+1}^{n}\left(2 \operatorname{Im} w_{j}\right)^{\lambda} d A\left(w_{1}\right) \cdots d A\left(w_{n}\right) .
\end{aligned}
$$

Denote by $\mathscr{A}_{\lambda}^{2}\left(M_{k}\right)$ the (weighted) Bergman subspace of $L^{2}\left(M_{k}, d V_{\lambda}^{k}\right)$. As always, the Bergman space is the subspace of the corresponding $L^{2}$-space which consists of all analytic functions.

The Cayley transform $p: \Pi \rightarrow \mathbb{D}$ is given by

$$
p(w)=\frac{w-i}{w+i}
$$

and the inverse Cayley transform $p^{-1}: \mathbb{D} \rightarrow \Pi$ acts by the rule

$$
p^{-1}(z)=i \frac{1+z}{1-z}
$$

Let id $: \mathbb{D} \rightarrow \mathbb{D}$ denote the identity map. The map $p_{k}: M_{k} \rightarrow$ $\mathbb{D}^{n}$ given by

$$
\begin{aligned}
& p_{k}(w) \\
& \quad=\left(\operatorname{id}\left(w_{1}\right), \ldots, \operatorname{id}\left(w_{k_{1}}\right), p\left(w_{k_{1}+1}\right), \ldots, p\left(w_{n}\right)\right)
\end{aligned}
$$

is obviously a biholomorphism.

It is easy to check directly that

$$
\begin{aligned}
& d V_{\lambda}(p(w))=\left|\prod_{j=k_{1}+1}^{n} \frac{2 i}{\left(w_{j}+i\right)^{2}}\right|^{\lambda+2} d V_{\lambda}^{k}(w), \\
& \quad=\left(\prod_{j=1}^{\lambda} \frac{1}{1-w_{j} \bar{\zeta}} \cdot \prod_{j=k_{1}+1}^{k_{1}} \frac{\left(w_{j}+i\right)\left(\bar{\zeta}_{j}-i\right)}{2 i\left(\bar{\zeta}_{j}-w_{j}\right)}\right)^{\lambda+2}, \\
& 1-p\left(\zeta_{j}\right)=\frac{2 i}{\zeta_{j}+i}, \quad j=k_{1}+1, \ldots, n,
\end{aligned}
$$

where $w, \zeta \in M_{k}$.

The map $p_{k}$ generates the operator

$$
U_{\lambda, k}: L^{2}\left(\mathbb{D}^{n}, d V_{\lambda}\right) \longrightarrow L^{2}\left(M_{k}, d V_{\lambda}^{k}\right)
$$

acting by the rule

$$
\begin{aligned}
\left(U_{\lambda, k} f\right)(w) & =\left(\prod_{j=k_{1}+1}^{n} p^{\prime}\left(w_{j}\right)\right)^{\lambda / 2+1}\left(f \circ p_{k}\right)(w) \\
& =\left(\prod_{j=k_{1}+1}^{n} \frac{2 i}{\left(w_{j}+i\right)^{2}}\right)^{\lambda / 2+1}\left(f \circ p_{k}\right)(w) .
\end{aligned}
$$

Let $f \in L^{2}\left(\mathbb{D}^{n}, d V_{\lambda}\right)$; changing the variables $z=p_{k}(w)$ and using (11) we have

$$
\begin{aligned}
& \|f\|_{L^{2}\left(\mathbb{D}^{n}, d V_{\lambda}\right)}^{2}=\int_{\mathbb{D}^{n}}|f(z)|^{2} d V_{\lambda}(z) \\
& \quad=\int_{M_{k}}\left|f \circ p_{k}(w)\right|^{2}\left|\prod_{j=k_{1}+1}^{n} \frac{2 i}{\left(w_{j}+i\right)^{2}}\right|^{\lambda+2} d V_{\lambda}^{k}(w) \\
& \quad=\left\|U_{\lambda, k} f\right\|_{L^{2}\left(M_{k}, d V_{\lambda}^{k}\right)}^{2} .
\end{aligned}
$$


The operator $U_{\lambda, k}$ is unitary; its inverse operator

$$
U_{\lambda, k}^{-1}: L^{2}\left(M_{k}, d V_{\lambda}^{k}\right) \longrightarrow L^{2}\left(\mathbb{D}^{n}, d V_{\lambda}\right)
$$

has the form

$$
\begin{aligned}
\left(U_{\lambda, k}^{-1} g\right)(z) & =\left(\prod_{j=k_{1}+1}^{n}\left(p_{k}^{-1}\right)^{\prime}(z)\right)^{\lambda / 2+1}\left(g \circ p_{k}^{-1}\right)(z) \\
& =\left(\prod_{j=k_{1}+1}^{n} \frac{2 i}{\left(1-z_{j}\right)^{2}}\right)^{\lambda / 2+1}\left(g \circ p_{k}^{-1}\right)(z) .
\end{aligned}
$$

It is obvious that $\mathscr{A}_{\lambda}^{2}\left(M_{k}\right)=U_{\lambda, k}\left(\mathscr{A}_{\lambda}^{2}\left(\mathbb{D}^{n}\right)\right)$; then the (weighted) Bergman space $\mathscr{A}_{\lambda}^{2}\left(M_{k}\right)$ is a closed subspace of $L^{2}\left(M_{k}, d V_{\lambda}^{k}\right)$. Changing the variables $z=p_{k}(z)$ and using (11), (12), and (13) we have that the (weighted) Bergman projection $B_{k, \lambda}$ of $L^{2}\left(M_{k}, d V_{\lambda}^{k}\right)$ onto $\mathscr{A}_{\lambda}^{2}\left(M_{k}\right)$ is given by

$$
\begin{aligned}
& \left(B_{k, \lambda} g\right)(w)=\left(U_{\lambda, k} B_{\mathbb{D}^{n}}^{\lambda} U_{\lambda, k}^{-1} f\right)(w) \\
& =\int_{M_{k}}\left(\prod_{j=k_{1}+1}^{n} \frac{(2 i)^{2}}{(1-p(\zeta))^{2}\left(w_{j}+i\right)^{2}}\right)^{\lambda / 2+1} g(\zeta) \\
& \cdot \overline{K_{\mathbb{D}^{n}}^{\lambda}\left(p_{k}(w), p_{k}(\zeta)\right)} d V_{\lambda}\left(p_{k}(\zeta)\right) \\
& =\int_{M_{k}} g(\zeta) \overline{K_{M_{k}}^{\lambda}(w, \zeta)} d V_{\lambda}^{k}(\zeta),
\end{aligned}
$$

where the (weighted) Bergman kernel has the form

$$
K_{M_{k}}^{\lambda}(w, \zeta)=\prod_{j=1}^{k_{1}} \frac{1}{\left(1-\overline{w_{j}} \zeta_{j}\right)^{\lambda+2}} \cdot \prod_{j=k_{1}+1}^{n} \frac{i^{\lambda+2}}{\left(\overline{w_{j}}-\zeta_{j}\right)^{\lambda+2}}
$$

\section{Representation of the Weighted Bergman Space on the Polydisk}

We present a construction of the Bargmann type transform $R_{\lambda}$ for the case of the polydisk, which maps isometrically the Bergman space onto an appropriate $L^{2}$ space and which allows us to factor the identity operator in the $L^{2}$ space and the Bergman projection of the polydisk. This scheme has been already successfully used, for example, in [2, 4-6]; we give here an analogous study for the polydisk.

We will use the multi-index notation. For an element $a=$ $\left(a_{1}, \ldots, a_{m}\right) \in \mathbb{C}^{m}$, let $|a|$ denote the sum

$$
|a|=\sum_{j=1}^{m} a_{j}
$$

Let $\alpha \in \mathbb{C}$ denote by $[\alpha]_{m}$ the vector $(\alpha, \ldots, \alpha) \in \mathbb{C}^{m}$. Introduce in $M_{k}$ the coordinates

$$
z_{j}=z_{j}^{\prime}=r_{j} t_{j},
$$

$$
r_{j} \in[0,1], t_{j} \in S^{1}, j=1, \ldots k_{1}
$$

$$
\begin{gathered}
z_{k_{1}+j}=\zeta_{j}=x_{j}+i y_{j}, \\
x_{j} \in \mathbb{R}, y_{j} \in \mathbb{R}_{+}, j=1, \ldots, k_{2} ; \\
z_{k_{1}+k_{2}+j}=\zeta_{j}^{\prime}=\rho_{j} e^{i \theta_{j}}, \\
\rho_{j} \in \mathbb{R}_{+}, \theta_{j} \in[0, \pi], j=1, \ldots, k_{3} .
\end{gathered}
$$

Under the identification

$$
\begin{aligned}
z & =\left(z_{1}, \ldots, z_{n}\right)=\left(z^{\prime}, \zeta, \zeta^{\prime}\right)=\left(r t, x+i y, \rho e^{i \theta}\right) \\
& =(t, x, \rho, r, y, \theta),
\end{aligned}
$$

we have

$$
\begin{aligned}
M_{k} & =\left([0,1] \times S^{1}\right)^{k_{1}} \times\left(\mathbb{R} \times \mathbb{R}_{+}\right)^{k_{2}} \times(\mathbb{R} \times[0, \pi])^{k_{3}} \\
& =G_{k} \times \Omega_{k},
\end{aligned}
$$

where

$$
\begin{aligned}
& G_{k}=\mathbb{T}^{k_{1}} \times \mathbb{R}^{k_{2}} \times \mathbb{R}_{+}^{k_{3}}, \\
& \Omega_{k}=[0,1]^{k_{1}} \times \mathbb{R}_{+}^{k_{2}} \times[0, \pi]^{k_{3}} .
\end{aligned}
$$

Let $(r, y, \theta) \in \Omega_{k}$, we define the measure

$$
\begin{aligned}
& d \eta(r, y, \theta) \\
& \quad=\prod_{j=1}^{k_{1}}\left(1-r_{j}\right)^{\lambda} r_{j} \prod_{j=1}^{k_{2}}\left(2 y_{j}\right)^{\lambda} \prod_{j=1}^{k_{3}} 2^{\lambda} \sin ^{\lambda} \theta_{j} d r d y d \theta .
\end{aligned}
$$

We have

$$
\begin{aligned}
& d V_{\lambda}^{k}(z)=d V_{\lambda}^{k}\left(z^{\prime}, \zeta, \zeta^{\prime}\right) \\
& =\left(\frac{\lambda+1}{\pi}\right)^{n} \frac{\left(\rho_{1} \cdots \rho_{k_{3}}\right)^{\lambda+1}}{i^{k_{1}} t_{1} \cdots t_{k_{1}}} d t d x d \rho d \eta(r, y, \theta) .
\end{aligned}
$$

Then we can write

$$
\begin{aligned}
L^{2}\left(M_{k}, d V_{\lambda}^{k}\right)= & L^{2}\left(\mathbb{T}^{k_{1}}\right) \otimes L^{2}\left(\mathbb{R}^{k_{2}}\right) \otimes L^{2}\left(\mathbb{R}_{+}^{k_{3}}\right) \\
& \otimes L^{2}\left(\Omega_{k},\left(\frac{1}{\pi^{n}}\right)(\lambda+1)^{n} d \eta\right),
\end{aligned}
$$

where

$$
\begin{aligned}
& L^{2}\left(\mathbb{T}^{k_{1}}\right)=\bigotimes_{j=1}^{k_{1}} L^{2}\left(S^{1}, \frac{d t_{j}}{i t_{j}}\right), \\
& L^{2}\left(\mathbb{R}^{k_{2}}\right)=\bigotimes_{j=1}^{k_{2}} L^{2}\left(\mathbb{R}, d x_{j}\right), \\
& L^{2}\left(\mathbb{R}_{+}^{k_{3}}\right)=\bigotimes_{j=1}^{k_{3}} L^{2}\left(\mathbb{R}_{+}, \rho_{j}^{\lambda+1} d \rho_{j}\right) .
\end{aligned}
$$


Consider the following three unitary operators:

(i) The discrete Fourier transform $\mathscr{F}: L^{2}\left(S^{1}, d t / i t\right) \rightarrow$ $\ell^{2}=\ell^{2}(\mathbb{Z})$ is given by

$$
\mathscr{F}: f \longmapsto c_{m}=\frac{1}{\sqrt{2 \pi}} \int_{S^{1}} f(t) t^{-m} \frac{d t}{i t} ;
$$

the inverse operator $\mathscr{F}^{-1}$ has the form

$$
\mathscr{F}^{-1}:\left\{c_{m}\right\}_{m \in \mathbb{Z}} \longmapsto f=\sum_{m \in \mathbb{Z}} c_{m} t^{m} .
$$

(ii) The Fourier integral transform $F: L^{2}(\mathbb{R}) \rightarrow L^{2}(\mathbb{R})$ is given by

$$
(F f)(u)=\frac{1}{\sqrt{2 \pi}} \int_{\mathbb{R}} e^{-i u x} f(x) d x ;
$$

the inverse operator $F^{-1}$ has the form

$$
\left(F^{-1} g\right)(x)=\frac{1}{\sqrt{2 \pi}} \int_{\mathbb{R}} e^{i x u} g(u) d u .
$$

(iii) The Mellin transform $M: L^{2}\left(\mathbb{R}_{+}, \rho^{\lambda+1}\right) \rightarrow L^{2}(\mathbb{R})$ is given by

$$
(M \psi)(\xi)=\frac{1}{\sqrt{2 \pi}} \int_{\mathbb{R}_{+}} \rho^{-i \xi+\lambda / 2} \psi(\rho) d \rho ;
$$

the inverse operator $M^{-1}$ has the form

$$
\left(M^{-1} \psi\right)(\rho)=\frac{1}{\sqrt{2 \pi}} \int_{\mathbb{R}} \rho^{i \xi-\lambda / 2-1} \psi(\xi) d \xi
$$

Let $\mathbb{Z}^{k_{1}}=\mathbb{Z} \times \cdots \times \mathbb{Z}$. Introduce the unitary operator

$$
\mathcal{U}_{\lambda, k}=\left(\frac{1}{\sqrt{\pi}}\right)^{n} \mathscr{F}_{\left(k_{1}\right)} \otimes F_{\left(k_{2}\right)} \otimes M_{\left(k_{3}\right)} \otimes I,
$$

acting from

$$
\begin{aligned}
L^{2}\left(M_{k}, d V_{\lambda}^{k}\right)= & L^{2}\left(\mathbb{T}^{k_{1}}\right) \otimes L^{2}\left(\mathbb{R}^{k_{2}}\right) \otimes L^{2}\left(\mathbb{R}_{+}^{k_{3}}\right) \\
& \otimes L^{2}\left(\Omega_{k},\left(\frac{1}{\pi^{n}}\right)(\lambda+1)^{n} d \eta\right)
\end{aligned}
$$

onto

$$
\begin{gathered}
\ell^{2}\left(\mathbb{Z}^{k_{1}}\right) \otimes L^{2}\left(\mathbb{R}^{k_{2}}\right) \otimes L^{2}\left(\mathbb{R}^{k_{3}}\right) \\
\otimes L^{2}\left(\Omega_{k},(\lambda+1)^{n} d \eta\right),
\end{gathered}
$$

where $\mathscr{F}_{\left(k_{1}\right)}=\mathscr{F} \otimes \cdots \otimes \mathscr{F}, F_{\left(k_{2}\right)}=F \otimes \cdots \otimes F, M_{\left(k_{3}\right)}=$ $M \otimes \cdots \otimes M$, and

$$
L^{2}\left(\mathbb{R}^{k_{3}}\right)=\bigotimes_{j=1}^{k_{3}} L^{2}\left(\mathbb{R}, d \rho_{j}\right)
$$

In the coordinates $z=\left(r t, x+i y, \rho e^{i \theta}\right)$ the Cauchy-Riemann equations have the form

$$
\begin{aligned}
& \frac{t_{j}}{2}\left(\frac{\partial}{\partial r_{j}}-\frac{t_{j}}{r_{j}} \frac{\partial}{\partial t_{j}}\right), \quad j=1, \ldots, k_{1}, \\
& \frac{1}{2}\left(\frac{\partial}{\partial x_{j}}+i \frac{\partial}{\partial y_{j}}\right), \quad j=1, \ldots, k_{2}, \\
& \left(\rho_{j} \frac{\partial}{\partial \rho_{j}}+i \frac{\partial}{\partial \theta_{j}}\right), \quad j=1, \ldots, k_{3} .
\end{aligned}
$$

Let $\left\{\psi_{q}\right\}_{q \in \mathbb{Z}^{k_{1}}} \in \ell^{2}\left(\mathbb{Z}^{k_{1}}, L^{2}\left(\mathbb{R}^{k_{2}}\right) \otimes L^{2}\left(\mathbb{R}^{k_{3}}\right) \otimes L^{2}\left(\Omega_{k},(\lambda+1)^{n} d \eta\right)\right)$. It straightforward to see that

$$
\begin{aligned}
& \left(\mathcal{U}_{\lambda} \frac{t_{j}}{2}\left(\frac{\partial}{\partial r_{j}}-\frac{t_{j}}{r_{j}} \frac{\partial}{\partial t_{j}}\right) \mathcal{U}_{\lambda}^{-1}\left\{\psi_{q}\right\}_{q \in \mathbb{Z}^{k_{1}}}\right)(m)(w) \\
& =\frac{1}{2}\left(\frac{\partial \psi_{m-\mathbf{1}_{j}}}{\partial r_{j}}-\frac{\left(m_{j}-1\right) \psi_{m-\mathbf{1}_{j}}}{r_{j}}\right)(w), \\
& \left(\mathcal{U}_{\lambda} \frac{1}{2}\left(\frac{\partial}{\partial x_{j}}+i \frac{\partial}{\partial y_{j}}\right) \mathcal{U}_{\lambda}^{-1}\left\{\psi_{q}\right\}_{q \in \mathbb{Z}^{k_{1}}}\right)(m)(w), k_{1}, \\
& =\frac{i}{2}\left(u_{j}+\frac{\partial}{\partial y_{j}}\right) \psi_{m}(w), \quad j=1, \ldots, k_{2}, \\
& \left(\mathcal{U}_{\lambda}\left(\rho_{j} \frac{\partial}{\partial \rho_{j}}+i \frac{\partial}{\partial \theta_{j}}\right) \mathcal{U}_{\lambda}^{-1}\left\{\psi_{q}\right\}_{q \in \mathbb{Z}^{k_{1}}}\right)(m)(w) \\
& =i\left(\left(\xi_{j}+\left(\frac{\lambda}{2}+1\right) i\right) I+\frac{\partial}{\partial \theta_{j}}\right) \psi_{m}(w),
\end{aligned}
$$

with $w=(u, \xi, r, y, \theta) \in \mathbb{R}^{k_{2}} \times \mathbb{R}^{k_{3}} \times \Omega_{k}$ and $m \in \mathbb{Z}^{k_{1}}$. Here $\mathbf{1}_{j}$ denotes the vector whose $j$ th entry is 1 and zero elsewhere. Thus the image $\mathscr{A}_{\lambda, k}^{1}=\mathscr{U}_{\lambda, k}\left(\mathscr{A}_{\lambda}^{2}\left(M_{k}\right)\right)$ consists of all sequences $\left\{\psi_{q}\right\}_{q \in \mathbb{Z}^{k_{1}}} \in \ell^{2}\left(\mathbb{Z}^{k_{1}}, L^{2}\left(\mathbb{R}^{k_{2}}\right) \otimes L^{2}\left(\mathbb{R}^{k_{3}}\right) \otimes\right.$ $\left.L^{2}\left(\Omega_{k},(\lambda+1)^{n} d \eta\right)\right)$ satisfying the equations

$$
\left(\frac{\partial}{\partial r_{j}}-\frac{m_{j}}{r_{j}} I\right) \psi_{m}=0 \text {, }
$$

$$
\begin{aligned}
& j=1, \ldots, k_{1}, \\
& \left(u_{j} I+\frac{\partial}{\partial y_{j}}\right) \psi_{m}=0,
\end{aligned}
$$

$$
j=1, \ldots, k_{2} \text {, }
$$

$\left(\left(\xi_{j}+\left(\frac{\lambda}{2}+1\right) i\right) I+\frac{\partial}{\partial \theta_{j}}\right) \psi_{m}=0$,

$$
j=1, \ldots, k_{3} \text {. }
$$


These equations are easy to solve; the general solution has the form

$$
\psi_{m}(u, \xi, r, y, \theta)=c_{m}^{0}(u, \xi) r^{m} e^{-|u y|-\left|\left(\xi+[1+\lambda / 2]_{k_{3}} i\right) \theta\right|}
$$

with $c_{m}^{0} \in L^{2}\left(\mathbb{R}^{k_{2}}\right) \otimes L^{2}\left(\mathbb{R}^{k_{3}}\right), m \in \mathbb{Z}^{k_{1}}$. The function $\psi_{m}$ has to be in $\left.L^{2}\left(\mathbb{R}^{k_{2}}\right) \otimes L^{2}\left(\mathbb{R}^{k_{3}}\right) \otimes L^{2}\left(\Omega_{k},(\lambda+1)^{n} d \eta\right)\right)$, which implies that its support on each variable $u_{j}, j=1, \ldots, k_{2}$, has to be in $\mathbb{R}_{+}$and each integer $m_{j}, j=1, \ldots, k_{1}$, has to be in $\mathbb{Z}_{+}=$ $\{0\} \cup \mathbb{N}$. That is,

$$
\begin{aligned}
\psi_{m} & (u, \xi, r, y, \theta) \\
& =\chi_{+}^{k_{2}}(u) \vartheta_{m}(u, \xi) c_{m}(u, \xi) r^{m} e^{-|u y|-\left|\left(\xi+[1+\lambda / 2]_{k_{3}} i\right) \theta\right|},
\end{aligned}
$$

where $m \in \mathbb{Z}_{+}^{k_{1}}=\mathbb{Z}_{+} \times \cdots \times \mathbb{Z}_{+}, c_{m} \in L^{2}\left(\mathbb{R}^{k_{2}}\right) \otimes L^{2}\left(\mathbb{R}^{k_{3}}\right), \chi_{+}^{k_{2}}$ is the characteristic function of $\mathbb{R}_{+}^{k_{2}}=\mathbb{R}_{+} \times \cdots \times \mathbb{R}_{+}$, and (see, e.g., formulas 3.251, 3.381, and 3.892 of [7])

$$
\begin{aligned}
\vartheta_{m} & (u, \xi) \\
= & \left((\lambda+1)^{n} \int_{\Omega_{k}} r^{2 m} e^{-2|u y|-2|\xi \theta|} d \eta(r, y, \theta)\right)^{-1 / 2} \\
= & \prod_{j=1}^{k_{1}}\left(\frac{2 \Gamma\left(m_{j}+2+\lambda\right)}{m_{j} ! \Gamma(2+\lambda)}\right)^{1 / 2} \\
& \cdot \prod_{j=1}^{k_{2}}\left(\frac{2 u_{j}^{\lambda+1}}{(\lambda+1) \Gamma(\lambda+1)}\right)^{1 / 2} \\
& \cdot \prod_{j=1}^{k_{3}} \frac{\left|\Gamma\left((\lambda+2) / 2+i \xi_{j}\right)\right|}{\sqrt{\pi} \Gamma(\lambda+2)^{1 / 2}} e^{\pi \xi_{j} / 2}
\end{aligned}
$$

with $u_{j} \geq 0, j=1, \ldots, k_{2}$. Moreover, we have

$$
\begin{aligned}
& \left\|\psi_{m}\right\|_{L^{2}\left(\mathbb{R}^{k_{2}}\right) \otimes L^{2}\left(\mathbb{R}^{k_{3}}\right) \otimes L^{2}\left(\Omega_{k},(\lambda+1)^{n} d \eta\right)}^{2} \chi_{\mathbb{R}^{k_{2}} \times \mathbb{R}^{k_{3}} \times \Omega_{k}} \chi_{+}^{k_{2}}(u) \vartheta_{m}^{2}(u, \xi) \\
& \cdot\left|c_{m}(u, \xi) r^{m} e^{-|u y|-\left|\left(\xi+[1+\lambda / 2]_{k_{3}} i\right) \theta\right|}\right|^{2} \\
& \cdot(\lambda+1)^{n} d u d \xi d \eta(r, y, \theta) \\
& =\int_{\mathbb{R}^{k_{2}} \times \mathbb{R}^{k_{3}}} \chi_{+}^{k_{2}}(u) \vartheta_{m}^{2}(u, \xi)\left|c_{m}(u, \xi)\right|^{2}(\lambda+1)^{n} \\
& \cdot \int_{\Omega_{k}} r^{2 m} e^{-2|u y|-2|\xi \theta|} d \eta(r, y, \theta) d u d \xi \\
& =\left\|c_{m}\right\|_{L^{2}\left(\mathbb{R}_{+}^{k_{2}}\right) \otimes L^{2}\left(\mathbb{R}^{k_{3}}\right)}^{2} .
\end{aligned}
$$

Lemma 1. The unitary operator $\mathscr{U}_{\lambda, k}=\left(1 /(\sqrt{\pi})^{n}\right) \mathscr{F}_{\left(k_{1}\right)} \otimes$ $F_{\left(k_{2}\right)} \otimes M_{\left(k_{3}\right)} \otimes I$ is an isometric isomorphism of the space $L^{2}\left(M_{k}, d V_{\lambda}^{k}\right)$ onto

$$
\ell^{2}\left(\mathbb{Z}^{k_{1}}, L^{2}\left(\mathbb{R}^{k_{2}}\right) \otimes L^{2}\left(\mathbb{R}^{k_{3}}\right) \otimes L^{2}\left(\Omega_{k},(\lambda+1)^{n} d \eta\right)\right),
$$

under which the Bergman space $\mathscr{A}_{\lambda}^{2}\left(M_{k}\right)$ is mapped onto

$$
\begin{aligned}
& \mathscr{A}_{\lambda, k}^{1}=\left\{\left\{\psi_{m}=\chi_{+}^{k_{2}}(u) \vartheta_{m}(u, \xi) c_{m}(u, \xi)\right.\right. \\
& \left.\cdot r^{m} e^{-|u y|-\left|\left(\xi+[1+\lambda / 2]_{k_{3}} i\right) \theta\right|}\right\}_{m \in \mathbb{Z}_{+}^{k_{1}}}: c_{m} \in L^{2}\left(\mathbb{R}_{+}^{k_{2}}\right) \\
& \left.\otimes L^{2}\left(\mathbb{R}^{k_{3}}\right)\right\} .
\end{aligned}
$$

Following [2] we introduce the isometric embedding $R_{0, k}$ acting from the space

$$
\ell^{2}\left(\mathbb{Z}_{+}^{k_{1}}, L^{2}\left(\mathbb{R}_{+}^{k_{2}}\right) \otimes L^{2}\left(\mathbb{R}^{k_{3}}\right)\right)
$$

onto

$$
\ell^{2}\left(\mathbb{Z}^{k_{1}}, L^{2}\left(\mathbb{R}^{k_{2}}\right) \otimes L^{2}\left(\mathbb{R}^{k_{3}}\right) \otimes L^{2}\left(\Omega_{k},(\lambda+1)^{n} d \eta\right)\right),
$$

by the rule

$$
\begin{aligned}
R_{0, k} & : c_{m}(u, \xi) \longmapsto \psi_{m} \\
& =\chi_{+}^{k_{2}}(u) \vartheta_{m}(u, \xi) c_{m}(u, \xi) r^{m} e^{-|u y|-\left|\left(\xi+[1+\lambda / 2]_{k_{3}} i\right) \theta\right|},
\end{aligned}
$$

where $c_{m}(u, \xi)$ is extended by $c_{m}(u, \xi)=0, u \in \mathbb{R}^{k_{2}} \backslash \mathbb{R}_{+}^{k_{2}}$.

The adjoint operator $R_{0, k}^{*}$ acting from

$$
\ell^{2}\left(\mathbb{Z}^{k_{1}}, L^{2}\left(\mathbb{R}^{k_{2}}\right) \otimes L^{2}\left(\mathbb{R}^{k_{3}}\right) \otimes L^{2}\left(\Omega_{k},(\lambda+1)^{n} d \eta\right)\right)
$$

onto $\ell^{2}\left(\mathbb{Z}_{+}^{k_{1}}, L^{2}\left(\mathbb{R}_{+}^{k_{2}}\right) \otimes L^{2}\left(\mathbb{R}^{k_{3}}\right)\right)$ has obviously the form

$$
\begin{gathered}
R_{0, k}^{*}: \psi_{m} \longmapsto c_{m}=(\lambda+1)^{n} \int_{\Omega_{k}} \psi_{m}(u, \xi, r, y, \theta) \\
\cdot r^{m} e^{-|u y|-\left|\left(\xi-[1+\lambda / 2]_{k_{3}} i \theta\right)\right|} d \eta(r, y, \theta) .
\end{gathered}
$$

It is easy to check that

$$
\begin{gathered}
R_{0, k}^{*} R_{0, k}=I: \ell^{2}\left(\mathbb{Z}_{+}^{k_{1}}, L^{2}\left(\mathbb{R}_{+}^{k_{2}}\right) \otimes L^{2}\left(\mathbb{R}^{k_{3}}\right)\right) \\
\longrightarrow \ell^{2}\left(\mathbb{Z}_{+}^{k_{1}}, L^{2}\left(\mathbb{R}_{+}^{k_{2}}\right) \otimes L^{2}\left(\mathbb{R}^{k_{3}}\right)\right), \\
R_{0, k} R_{0, k}^{*}=B: \ell^{2}\left(\mathbb{Z}^{k_{1}}, L^{2}\left(\mathbb{R}^{k_{2}}\right) \otimes L^{2}\left(\mathbb{R}^{k_{3}}\right)\right. \\
\left.\otimes L^{2}\left(\Omega_{k},(\lambda+1)^{n} d \eta\right)\right) \longrightarrow \mathscr{A}_{\lambda, k}^{1},
\end{gathered}
$$

where $B=\mathcal{U}_{\lambda, k} B_{M_{k}}^{\lambda} \mathcal{U}_{\lambda, k}^{-1}$ is the orthogonal projection of

$$
\ell^{2}\left(\mathbb{Z}^{k_{1}}, L^{2}\left(\mathbb{R}^{k_{2}}\right) \otimes L^{2}\left(\mathbb{R}^{k_{3}}\right) \otimes L^{2}\left(\Omega_{k},(\lambda+1)^{n} d \eta\right)\right)
$$

onto $\mathscr{A}_{\lambda, k}^{1}$.

The operator $R_{\lambda, k}=R_{0, k}^{*} \mathcal{U}_{\lambda, k}$ maps the space $L^{2}\left(M_{k}, d V_{\lambda}^{k}\right)$ onto

$$
\ell^{2}\left(\mathbb{Z}_{+}^{k_{1}}, L^{2}\left(\mathbb{R}_{+}^{k_{2}}\right) \otimes L^{2}\left(\mathbb{R}^{k_{3}}\right)\right),
$$

and the restriction

$$
\begin{aligned}
& \left.R_{\lambda, k}\right|_{\mathscr{A}_{\lambda}^{2}\left(M_{k}\right)}: \mathscr{A}_{\lambda}^{2}\left(M_{k}\right) \\
& \quad \longrightarrow \ell^{2}\left(\mathbb{Z}_{+}^{k_{1}}, L^{2}\left(\mathbb{R}_{+}^{k_{2}}\right) \otimes L^{2}\left(\mathbb{R}^{k_{3}}\right)\right)
\end{aligned}
$$


is an isometric isomorphism. The adjoint operator

$$
\begin{aligned}
R_{\lambda, k}^{*} & =\mathscr{U}_{\lambda, k}^{*} R_{0, k}: \ell^{2}\left(\mathbb{Z}_{+}^{k_{1}}, L^{2}\left(\mathbb{R}_{+}^{k_{2}}\right) \otimes L^{2}\left(\mathbb{R}^{k_{3}}\right)\right) \\
& \longrightarrow \mathscr{A}_{\lambda}^{2}\left(M_{k}\right)
\end{aligned}
$$

is an isometric isomorphism.

Remark 2. We have

$$
\begin{aligned}
R_{k, \lambda} R_{k, \lambda}^{*} & =I: \ell^{2}\left(\mathbb{Z}_{+}^{k_{1}}, L^{2}\left(\mathbb{R}_{+}^{k_{2}}\right) \otimes L^{2}\left(\mathbb{R}^{k_{3}}\right)\right) \\
& \longrightarrow \ell^{2}\left(\mathbb{Z}_{+}^{k_{1}}, L^{2}\left(\mathbb{R}_{+}^{k_{2}}\right) \otimes L^{2}\left(\mathbb{R}^{k_{3}}\right)\right), \\
R_{k, \lambda}^{*} R_{k, \lambda} & =B_{M_{k}}^{\lambda}: L^{2}\left(M_{k}, d V_{\lambda}^{k}\right) \longrightarrow \mathscr{A}_{\lambda}^{2}\left(M_{k}\right) .
\end{aligned}
$$

Theorem 3. The isometric isomorphism $R_{\lambda, k}^{*}: \ell^{2}\left(\mathbb{Z}_{+}^{k_{1}}, L^{2}\left(\mathbb{R}_{+}^{k_{2}}\right) \otimes\right.$ $\left.L^{2}\left(\mathbb{R}^{k_{3}}\right)\right) \rightarrow \mathscr{A}_{\lambda}^{2}\left(M_{k}\right)$ is given by

$$
\begin{aligned}
& R_{\lambda, k}^{*}:\left\{c_{m}\right\}_{m \in \mathscr{Z}_{+}^{k_{1}}} \longmapsto \psi\left(z^{\prime}, \zeta, \zeta^{\prime}\right)=\left(\frac{1}{\sqrt{2}}\right)^{n} \\
& \cdot \sum_{m \in \mathbb{Z}_{+}^{k_{1}}} \int_{\mathbb{R}_{+}^{k_{2}} \times \mathbb{R}^{k_{3}}} \vartheta_{m}(u, \xi) c_{m}(u, \xi)\left(z^{\prime}\right)^{m} \\
& \cdot e^{i|u \zeta|}\left(\zeta^{\prime}\right)^{i \xi-[1+\lambda / 2]_{k_{3}}} d u d \xi .
\end{aligned}
$$

Proof. Let $\left\{c_{m}\right\}_{m \in \mathbb{Z}_{+}^{k_{1}}}$ be a sequence of functions in $\ell^{2}\left(\mathbb{Z}_{+}^{k_{1}}\right.$, $\left.L^{2}\left(\mathbb{R}_{+}^{k_{2}}\right) \otimes L^{2}\left(\mathbb{R}^{k_{3}}\right)\right)$; then we have

$$
\begin{gathered}
\left(\mathcal{U}_{\lambda, k}^{*} R_{0, k}\left\{c_{m}\right\}_{m \in \mathbb{Z}_{+}^{k_{1}}}\right)(u, \xi, r, y, \theta)=\left(\frac{1}{\sqrt{2}}\right)^{n} \\
\cdot \sum_{m \in \mathbb{Z}_{+}^{k_{1}}} \int_{\mathbb{R}^{k_{2} \times \mathbb{R}^{k_{3}}}}\left(R_{0, k}\left\{c_{q}\right\}_{q \in \mathbb{Z}_{+}^{k_{1}}}\right)(m)(u, \xi) \\
\cdot t^{m} e^{|x u|} \rho^{i \xi-[1+\lambda / 2]_{k_{3}}} d u d \xi=\left(\frac{1}{\sqrt{2}}\right)^{n} \\
\cdot \sum_{m \in \mathbb{Z}_{+}^{k_{1}}} \int_{\mathbb{R}_{+}^{k_{2}} \times \mathbb{R}^{k_{3}}} \vartheta_{m}(u, \xi) c_{m}(u, \xi)(r t)^{m} \\
\cdot e^{i|u(x+i y)|}\left(\rho e^{i \theta}\right)^{i \xi-[1+\lambda / 2]_{k_{3}}} d u d \xi,
\end{gathered}
$$

which proves the theorem.

Corollary 4. The inverse isomorphism $R_{\lambda, k}: \mathscr{A}_{\lambda}^{2}\left(M_{k}\right) \rightarrow$ $\ell^{2}\left(\mathbb{Z}_{+}^{k_{1}}, L^{2}\left(\mathbb{R}_{+}^{k_{2}}\right) \otimes L^{2}\left(\mathbb{R}^{k_{3}}\right)\right)$ is given by

$$
\begin{gathered}
R_{\lambda, k}: \psi \longmapsto\left\{\left(\frac{1}{\sqrt{2}}\right)^{n} \vartheta_{m}(u, \xi) \int_{M_{k}} \psi\left(z^{\prime}, \zeta, \zeta^{\prime}\right)\right. \\
\left.\cdot{\overline{z^{\prime}}}^{m} e^{|-i u \bar{\zeta}| \bar{\zeta}^{\left(-i \xi-[1+\lambda / 2]_{k_{3}}\right)}} d V_{\lambda}^{k}\left(z^{\prime}, \zeta, \zeta^{\prime}\right)\right\}_{m \in \mathbb{Z}_{+}^{k_{1}}}
\end{gathered}
$$

\section{Toeplitz Operators and Maximal Commutative Subgroups of Biholomorphisms}

One of the main results of [8] gives a classification of the maximal Abelian subgroups of biholomorphisms of the unit ball $\mathbb{B}^{n}$. In particular for the unit disk $\mathbb{D}$ or the upper halfplane $\Pi$, we have three different types of these subgroups.

The group of biholomorphisms Aut $(\mathbb{D})$ can be described as follows. Denote by $\mathrm{SU}(1,1)$ the special unitary group defined as the set of matrices $A$ of dimension $2 \times 2$ such that $\operatorname{det} A=1$ and $A^{*} E A=E$, where

$$
E=\left(\begin{array}{cc}
1 & 0 \\
0 & -1
\end{array}\right) \text {. }
$$

We have that $\operatorname{Aut}(\mathbb{D})$ is realized by the action of $\operatorname{SU}(1,1)$ given by

$$
\begin{aligned}
& \mathrm{SU}(1,1) \times \mathbb{D} \longrightarrow \mathbb{D} \\
& \left(\begin{array}{ll}
a & b \\
c & d
\end{array}\right) \cdot z=\frac{a z+b}{c z+d} .
\end{aligned}
$$

Definition 5. Let $G$ be a Lie group. A maximal connected Abelian subgroup $H$ of $G$ is a Lie subgroup of $G$ that satisfies the following:

(i) $H$ is connected Abelian.

(ii) If $H_{1}$ is a connected Abelian Lie subgroup of $G$ such that $H \subset H_{1}$, then $H=H_{1}$.

Definition 6. Let $M$ and $M^{\prime}$ be connected complex manifolds. If $H$ and $H^{\prime}$ are subgroups of $\operatorname{Aut}(M)$ and $\operatorname{Aut}\left(M^{\prime}\right)$, respectively, we will say that $(H, M)$ and $\left(H^{\prime}, M^{\prime}\right)$ are analytically equivalent if there is a biholomorphism $\phi: M^{\prime} \rightarrow M$ such that $H^{\prime}=\varphi^{-1} H \varphi$.

It is proved in [8] that every maximal connected Abelian subgroup of $\operatorname{Aut}(\mathbb{D})$ is analytically equivalent to one of the following three groups, while neither two from the list are analytically equivalent.

The elliptic group of biholomorphisms of the unit disk $\mathbb{D}$ is isomorphic to $S^{1}$ with the following group action:

$$
t: z \in \mathbb{D} \longmapsto t z \in \mathbb{D},
$$

for each $t \in S^{1}$.

The parabolic group of biholomorphisms of the upper half-plane $\Pi$ is isomorphic to $\mathbb{R}$ with the following group action:

$$
x: z \in \Pi \longmapsto x+z \in \Pi,
$$

for each $x \in \mathbb{R}$.

The hyperbolic group of biholomorphisms of the upper half-plane $\Pi$ is isomorphic to $\mathbb{R}_{+}$with the following group action:

$$
\rho: z \in \Pi \longmapsto \rho z \in \Pi
$$

for each $\rho \in \mathbb{R}_{+}$. 
Now we turn our attention to the case of the polydisk. We have [3] that $\operatorname{Aut}\left(\mathbb{D}^{n}\right)$ is realized by the action of $\operatorname{SU}(1,1)^{n}$ extended by the $n$th symmetric group. Consider the Lie group

$$
\Omega_{k}=\mathbb{T}^{k_{1}} \times \mathbb{R}^{k_{2}} \times \mathbb{R}_{+}^{k_{3}}
$$

acting on $M_{k}=\mathbb{D}^{k_{1}} \times \Pi^{k_{2}} \times \Pi^{k_{3}}$ by

$$
\begin{aligned}
(t, x, \rho): z & =\left(z^{\prime}, \zeta, \zeta^{\prime}\right) \in M_{k} \longmapsto\left(t z^{\prime}, x+\zeta, \rho \zeta^{\prime}\right) \\
& \in M_{k},
\end{aligned}
$$

for each $(t, x, \rho) \in \Omega_{k}$. It is evident that this group is analytically equivalent to a maximal Abelian subgroup of $\mathrm{SU}(1,1)^{n}$ for each $k=\left(k_{1}, k_{2}, k_{3}\right)$. Therefore we have $(n+$ $1)(n+2) / 2$ different types of maximal Abelian subgroups of biholomorphisms of $\mathbb{D}^{n}$.

We have that a function $a(z), z=\left(r t, x+i y, \rho e^{i \theta}\right) \in M_{k}$ is invariant with respect to the action of $\Omega_{k}$ if and only if $a(z)=a(r, y, \theta)$. Hence making use of the results of Section 3 we can give a spectral representation of the Toeplitz operators with symbols invariant under the action of a maximal Abelian subgroup of biholomorphisms.

Theorem 7. Let $a=a(r, y, \theta)$ be a bounded measurable function. Then the Toeplitz operator $T_{a}$ acting on $\mathscr{A}_{\lambda}^{2}\left(M_{k}\right)$ is unitary equivalent to the multiplication operator $\gamma_{a, k} I=$ $R_{\lambda, k} T_{a} R_{\lambda, k}^{*}$. The sequence $\gamma_{a, k}=\left\{\gamma_{a, k}(m, u, \xi)\right\}_{m \in \mathbb{Z}_{k_{1}}}$, where $u \in \mathbb{R}_{+}^{k_{2}}, \xi \in \mathbb{R}^{k_{3}}$, is given by

$$
\begin{aligned}
& \gamma_{a, k}(m, u, \xi)=(\lambda+1)^{n} \vartheta_{m}^{2}(u, \xi) \\
& \cdot \int_{\Omega_{k}} a(r, y, \theta) r^{2 m} e^{-2(|u y|+|\xi \theta|)} d \eta(r, y, \theta) .
\end{aligned}
$$

Proof. The operator $R_{\lambda, k}$ defined in Section 3 factors the identity operator $I$ and the Bergman projection $B_{M_{k}}^{\lambda}$ (Remark 2); then we have

$$
\begin{aligned}
R_{\lambda, k} T_{a} R_{\lambda, k}^{*} & =R_{\lambda, k} B_{M_{k}}^{\lambda} a B_{M_{k}}^{\lambda} R_{\lambda, k}^{*} \\
& =\left(R_{\lambda, k} R_{\lambda, k}^{*}\right) R_{\lambda, k} a R_{\lambda, k}^{*}\left(R_{\lambda, k} R_{\lambda, k}^{*}\right) \\
& =R_{0, k}^{*} \mathcal{U}_{\lambda, k} a(r, y, \theta) \mathcal{U}_{\lambda, k}^{*} R_{0, k} \\
& =R_{0, k}^{*} a(r, y, \theta) R_{0, k} .
\end{aligned}
$$

Let $\left\{c_{m}(u, \xi)\right\}_{m \in \mathbb{Z}_{+}^{k_{1}}}$ be a sequence in $\ell^{2}\left(\mathbb{Z}_{+}^{k_{1}}, L^{2}\left(\mathbb{R}_{+}^{k_{2}}\right) \otimes\right.$ $\left.L^{2}\left(\mathbb{R}^{k_{3}}\right)\right)$. Theorem 3 and Corollary 4 give an integral representation of the operators $R_{\lambda, k}$ and $R_{\lambda, k}^{*}$, respectively; then we have

$$
\begin{gathered}
R_{\lambda, k} T_{a} R_{\lambda, k}^{*}\left\{c_{m}(u, \xi)\right\}_{m \in \mathbb{Z}_{+}^{k_{1}}}=R_{0, k}^{*}\left\{a(r, y, \theta) \chi_{+}^{k_{2}}(u)\right. \\
\left.\cdot \vartheta_{m}(u, \xi) c_{m}(u, \xi) r^{m} e^{-|u y|-\left|\left(\xi+[1+\lambda / 2]_{k_{3}} i\right) \theta\right|}\right\}_{m \in \mathbb{Z}_{+}^{k_{1}}}
\end{gathered}
$$

$$
\begin{aligned}
& =\left\{(\lambda+1)^{n} \vartheta_{m}^{2}(u, \xi) c_{m}(u, \xi)\right. \\
& \left.\cdot \int_{\Omega_{k}} a(r, y, \theta) r^{2 m} e^{-2(|u y|+|\xi \theta|)} d \eta(r, y, \theta)\right\}_{m \in \mathbb{Z}_{+}^{k_{1}}} \\
& =\left\{\gamma_{a, k}(m, u, \xi) c_{m}(u, \xi)\right\}_{m \in \mathbb{Z}_{+}^{k_{1}}} .
\end{aligned}
$$

\section{The Moment Map}

Let $(M, \omega)$ be a symplectic manifold, let $G$ be a Lie group, and let $\mathfrak{g}$ be the Lie algebra of $G$. Denote by $\operatorname{Sympl}(M, \omega)$ the set of all symplectomorphisms of $(M, \omega)$. The action

$$
\begin{gathered}
\psi: G \longrightarrow S_{M} \\
g \longmapsto \psi_{g}
\end{gathered}
$$

is called a symplectic action if $\psi_{g} \in \operatorname{Sympl}(M, \omega)$ for all $g \in$ $G$. In this case we say that $G$ acts by symplectomorphisms.

A symplectic action $\psi: G \rightarrow \operatorname{Sympl}(M, \omega)$ is a Hamiltonian action if there exists a map

$$
\mu: M \longrightarrow \mathfrak{g}^{*}
$$

satisfying the following:

(1) For each $X \in \mathfrak{g}$, let

$$
\begin{aligned}
\mu^{X}: M & \longrightarrow \mathbb{R}, \\
p & \longmapsto\langle\mu(p), X\rangle .
\end{aligned}
$$

Let $X^{\sharp}$ be the vector field on $M$ generated by the oneparameter subgroup $\left\{e^{t X}: t \in \mathbb{R}\right\} \subset G$; then $\mu^{X}$ is a Hamiltonian function for the vector field $X^{\sharp}$; that is,

$$
i_{X^{\sharp}} \omega=d \mu^{X} \text {. }
$$

(2) $\mu$ is equivariant with respect to the action $\psi$ and the coadjoint action $\mathrm{Ad}^{*}$ of $G$ on $\mathfrak{g}^{*}$; that is,

$$
\mu \circ \psi_{g}=\operatorname{Ad}^{*} \circ \mu \text {, }
$$

for all $g \in G$.

The vector $(M, \omega, G, \mu)$ is called a Hamiltonian $G$-space and $\mu$ is a moment map.

In what follows we will suppose that $G$ is an Abelian Lie group. It is known that the Lie algebra of a connected Lie group is Abelian if and only if its Lie algebra is Abelian. Therefore if $G$ is Abelian we have that $\exp (X+Y)=$ $\exp (X) \exp (Y)$, with $X, Y \in \mathfrak{g}$ (see [9, Proposition 3.51]). A direct calculation shows the following.

Lemma 8. Let $G$ be a connected Abelian Lie group. If $X_{1}, \ldots, X_{n} \in \mathfrak{g}$, then

$$
\left(a_{1} X_{1}+\cdots+a_{n} X_{n}\right)^{\sharp}=a_{1} X_{1}^{\sharp}+\cdots+a_{n} X_{n}^{\sharp},
$$

where $a_{1}, \ldots, a_{n}$ are real numbers. 
Moreover, making use of the linearity of the symplectic form $\omega$ we can state the following result.

Proposition 9. Let $G$ be a connected Abelian Lie group which acts by symplectomorphisms on the symplectic manifold $(M, \omega)$. Let $X_{1}, \ldots, X_{n}$ be a basis of $\mathfrak{g}$. If $\mu: M \rightarrow \mathfrak{g}^{*}$ is a map such that $\mu^{X_{j}}$ is a Hamiltonian function of $X_{j}^{\sharp}, j=1, \ldots, n$, then

$$
i_{X^{\sharp}} \omega=d \mu^{X},
$$

for each $X \in \mathfrak{g}$.

If $G$ is Abelian, then the conjugate action of $G$ on $G$ is the trivial action. This implies that the coadjoint action $\mathrm{Ad}_{g}^{*}$ of $\mathfrak{g}^{*}$ on $\mathfrak{g}^{*}$ is the identity map for all $g \in G$. Consequently, we can restate condition (2) as follows:

(2) $\mu$ is invariant with respect to the action $\psi$; that is,

$$
\mu \circ \psi_{g}=\mu
$$

for all $g \in G$.

\section{The Moment Map of the Polydisk}

Let $\left(\mathbb{D}^{n}, \omega\right)$ be the symplectic manifold where

$$
\omega=\sum_{j=1}^{n} \frac{1}{\pi} \frac{d x_{j} \wedge d y_{j}}{\left(1-\left(x_{j}^{2}+y_{j}^{2}\right)\right)^{2}}=\sum_{j=1}^{k_{1}} \frac{d \overline{z_{j}} \wedge d z_{j}}{2 \pi i\left(1-\left|z_{j}\right|^{2}\right)^{2}} .
$$

For each $k=\left(k_{1}, k_{2}, k_{3}\right)$ consider the symplectic manifold $\left(M_{k}, \omega^{\prime}\right)$ where

$$
\begin{aligned}
\omega^{\prime}= & \sum_{j=1}^{k_{1}} \frac{1}{\pi} \frac{d x_{j} \wedge d y_{j}}{\left(1-\left(x_{j}^{2}+y_{j}^{2}\right)\right)^{2}}+\sum_{j=k_{1}+1}^{n} \frac{1}{\pi} \frac{d x_{j} \wedge d y_{j}}{\left(2 y_{j}\right)^{2}} \\
= & \sum_{j=1}^{k_{1}} \frac{\overline{d z_{j}^{\prime}} \wedge d z_{j}^{\prime}}{2 \pi i\left(1-\left|z_{j}^{\prime}\right|^{2}\right)^{2}}+\sum_{j=1}^{k_{2}} \frac{d \overline{\zeta_{j}} \wedge d \zeta_{j}}{2 \pi i\left(2 \operatorname{Im} \zeta_{j}\right)^{2}} \\
& +\sum_{j=1}^{k_{3}} \frac{d \overline{\zeta_{j}^{\prime}} \wedge d \zeta_{j}^{\prime}}{2 \pi i\left(2 \operatorname{Im} \zeta^{\prime}\right)^{2}} .
\end{aligned}
$$

It is easy to check directly that the map

$$
\begin{aligned}
p_{k}: w & \in M_{k} \longrightarrow\left(w_{1}, \ldots w_{k_{1}}, p\left(w_{k_{1}+1}\right), \ldots, p\left(w_{n}\right)\right) \\
& \in \mathbb{D}^{n}
\end{aligned}
$$

where $p$ is the Cayley transform defined by 1 , is a symplectomorphism of $M_{k}$ onto the polydisk $\mathbb{D}^{n}$; that is, $\omega^{\prime}=p_{k}^{*} \omega$.

Let $G_{k}$ denote the Lie group $\mathbb{T}^{k_{1}} \times \mathbb{R}^{k_{2}} \times \mathbb{R}_{+}^{k_{3}}$. The Lie algebra $\mathfrak{g}_{k}$ of this group is isomorphic to $\mathbb{R}^{n}$; then $\mathfrak{g}_{k}^{*} \cong \mathbb{R}^{n}$. Let $\psi$ be the action of $G_{k}$ onto $M_{k}$ defined by

$$
\begin{aligned}
\psi_{(t, x, \rho)}: z & =\left(z^{\prime}, \zeta, \zeta^{\prime}\right) \in M_{k} \longmapsto\left(t z^{\prime}, x+\zeta, \rho \zeta^{\prime}\right) \\
& \in M_{k} .
\end{aligned}
$$

We note that for each $(t, x, \rho) \in G_{k}$ the map $\psi_{(t, x, \rho)}$ is a diffeomorphism; moreover we have

$$
\begin{aligned}
\psi_{(t, x, \rho)}^{*} \omega^{\prime}= & \sum_{j=1}^{k_{1}}\left|\frac{\partial \psi_{(t, x, \rho)}}{\partial z_{j}^{\prime}}\right|^{2} \frac{\overline{d z_{j}^{\prime}} \wedge d z_{j}^{\prime}}{2 \pi i\left(1-\left|t_{j} z_{j}^{\prime}\right|^{2}\right)^{2}} \\
& +\sum_{j=1}^{k_{2}}\left|\frac{\partial \psi_{(t, x, \rho)}}{\partial \zeta_{j}}\right|^{2} \frac{d \overline{\zeta_{j}} \wedge d \zeta_{j}}{2 \pi i\left(2 \operatorname{Im}\left(x_{j}+\zeta_{j}\right)\right)^{2}} \\
& +\sum_{j=1}^{k_{3}}\left|\frac{\partial \psi_{(t, x, \rho)}}{\partial \zeta_{j}^{\prime}}\right|^{2} \frac{d \overline{\zeta_{j}^{\prime}} \wedge d \zeta_{j}^{\prime}}{2 \pi i\left(2 \operatorname{Im} \rho_{j} \zeta^{\prime}\right)^{2}} \\
= & \sum_{j=1}^{k_{1}}\left|t_{j}^{2}\right| \frac{d \overline{z_{j}^{\prime}} \wedge d z_{j}^{\prime}}{2 \pi i\left(1-\left|t_{j} z_{j}^{\prime}\right|^{2}\right)^{2}} \\
& +\sum_{j=1}^{k_{2}} \frac{d \overline{\zeta_{j}} \wedge d \zeta_{j}}{2 \pi i\left(2 \operatorname{Im}\left(x_{j}+\zeta_{j}\right)\right)^{2}} \\
& +\sum_{j=1}^{k_{3}} \rho_{j}^{2} \frac{d \overline{\zeta_{j}^{\prime}} \wedge d \zeta_{j}^{\prime}}{2 \pi i\left(2 \operatorname{Im} \rho_{j} \zeta^{\prime}\right)^{2}}=\omega^{\prime}
\end{aligned}
$$

which implies that $\psi$ is a symplectic action.

We introduce the functions $f_{j}: M_{k} \rightarrow \mathbb{R}, j=1, \ldots, n$, given by

$$
\begin{aligned}
& f_{j}(z)=\frac{1}{2 \pi\left(1-\left(x_{j}^{2}+y_{j}^{2}\right)\right)}, \quad j=1, \ldots, k_{1}, \\
& f_{j}(z)=-\frac{1}{4 \pi y_{j}}, \quad j=k_{1}+1, \ldots, n-k_{3}, \\
& f_{j}(z)=-\frac{x_{j}}{4 \pi y_{j}}, \quad j=n-k_{3}+1, \ldots, n .
\end{aligned}
$$

Lemma 10. The map $\mu: M_{k} \rightarrow \mathbb{R}^{m}$ defined by

$$
\mu(z)=\left(f_{1}(z), \ldots, f_{m}(z)\right)
$$

is a moment map for the symplectic action $\psi$ of $G_{k}$ into $\left(M_{k}, \omega^{\prime}\right)$; that is, $\left(M_{k}, \omega^{\prime}, G_{k}, \mu\right)$ is a Hamiltonian $G_{k}$-space.

Proof. Let $X_{1}, \ldots, X_{n}$ be the standard basis of $\mathfrak{g}_{k} \cong \mathbb{R}^{n}$. The vector fields $X_{1}^{\sharp}, \ldots, X_{n}^{\sharp}$ over $M_{k}$ generated by the the elements of this basis are given by

$$
\begin{aligned}
& X_{j}^{\sharp}=-y_{j} \frac{\partial}{\partial x_{j}}+x_{j} \frac{\partial}{\partial y_{j}}, \quad j=1, \ldots, k_{1} \\
& X_{j}^{\sharp}=\frac{\partial}{\partial x_{j}}, \quad j=k_{1}+1, \ldots, k_{3}-n \\
& X_{j}^{\sharp}=x_{j} \frac{\partial}{\partial x_{j}}+y_{j} \frac{\partial}{\partial y_{j}}, \quad j=k_{3}-n+1, \ldots, n .
\end{aligned}
$$


We observe that

$$
\begin{aligned}
& i_{X_{j}} \omega^{\prime}(z) \\
& =\frac{1}{\pi} \frac{d x_{j} \wedge d y_{j}}{\left(1-\left(x_{j}^{2}+y_{j}^{2}\right)\right)^{2}}\left(-\left.y_{j} \frac{\partial}{\partial x_{j}}\right|_{z}+\left.x_{j} \frac{\partial}{\partial y_{j}}\right|_{z}, \cdot\right) \\
& =-\frac{1}{\pi} \frac{y_{j} d y_{j}+x_{j} d x_{j}}{\left(1-\left(x_{j}^{2}+y_{j}^{2}\right)\right)^{2}}=d f_{j}(z), \quad j=1, \ldots, k_{1}, \\
& i_{X_{j}} \omega^{\prime}(z)=\frac{1}{\pi} \frac{d x \wedge d y_{j}}{\left(2 y_{j}\right)^{2}}\left(\left.\frac{\partial}{\partial x_{j}}\right|_{z}, \cdot\right)=\frac{1}{\pi} \frac{d y_{j}}{\left(2 y_{j}\right)^{2}} \\
& =d f_{j}(z), \quad j=k_{1}+1, \ldots, n-k_{3}, \\
& i_{X_{j}} \omega^{\prime}(z)=\frac{1}{\pi} \frac{d x_{s_{j}} \wedge d y_{j}}{\left(2 y_{j}\right)^{2}}\left(\left.x_{j} \frac{\partial}{\partial x_{j}}\right|_{z}+\left.y_{j} \frac{\partial}{\partial y_{j}}\right|_{z}, \cdot\right) \\
& =\frac{1}{\pi} \frac{x_{j} d y_{j}-y_{j} d x_{j}}{\left(2 y_{j}\right)^{2}}=d f_{j}(z),
\end{aligned}
$$

with $z \in M_{k}$. Hence Lemma 8 implies that

$$
i_{X} \omega^{\prime}=d \mu^{X}
$$

for all $X \in \mathfrak{h}$. It is easy to check directly that each function $f_{j}$, $j=1, \ldots, n$, is invariant under the action of $\psi$; consequently $\mu$ is invariant under this action too. Therefore $\mu$ is a moment map.

Our aim is to give a moment map for the action of each Abelian subgroup of biholomorphisms of the polydisk. Let $H$ be a Lie subgroup of dimension $m \leq n$ of $G_{k}$; then the Lie algebra $\mathfrak{h}$ of $H$ is a Lie subalgebra of $\mathfrak{g}_{k}$, which implies that $\mathfrak{h}^{*}$ is a subspace of $\mathfrak{g}_{k}^{*}$. Let $\pi: \mathfrak{g}_{k}^{*} \rightarrow \mathfrak{h}^{*}$ be the natural projection and consider the symplectic action of $H$ into $M_{k}$ as the restricted action $\left.\psi\right|_{H}$.

Theorem 11. The map $\mu_{H}=\pi \circ \mu: M_{k} \rightarrow \mathfrak{h}^{*}$ is a moment map for the symplectic action $\left.\psi\right|_{H}$ of $H$ into $\left(M_{k}, \omega^{\prime}\right)$; that is, $\left(M_{k}, \omega^{\prime}, H, \mu_{H}\right)$ is a Hamiltonian H-space.

Proof. Let $Y_{1}, \ldots, Y_{k}$ be a basis of $\mathfrak{h}$; we can write $Y_{j}=a_{1 j} X_{1}+$ $\cdots+a_{n j} X_{n}, j=1, \ldots, k$, as a linear combination of the standard basis $X_{1}, \ldots, X_{n}$. We observe

$$
\begin{aligned}
i_{Y_{j}^{\sharp}} \omega & =a_{1 j} i_{X_{1}^{\sharp}} \omega+\cdots+a_{n j} i_{X_{n}^{\sharp}} \omega \\
& =a_{1 j} d f_{1}+\cdots+a_{n j} d f_{n}=d \mu_{H}^{Y_{j}} .
\end{aligned}
$$

Using Proposition 9 we have that $i_{Y^{\sharp}} \omega=d \mu^{Y}$ for every $Y$ in $\mathfrak{h}$. Since it is immediate that $\mu_{H}$ is invariant under the action, we conclude that it is a moment map.

\section{Symbol Classes Generated by the Moment Map}

In this section we introduce a class of bounded measurable functions $\widetilde{S}_{H}$ over the polydisk associated with each connected Abelian Lie subgroup of biholomorphisms $H$ of $G_{k}=\mathbb{T}^{k_{1}} \times \mathbb{R}^{k_{2}} \times \mathbb{R}_{+}^{k_{3}}$ which are a group of isometries over $\left(M_{k}, \omega^{\prime}, H, \mu_{H}\right)$. Moreover, we have that the $C^{*}$-algebra generated by Toeplitz operators with symbols in $\widetilde{S}_{H}$ is commutative.

We consider a connected Abelian subgroup $H$ of $G_{k}=$ $\mathbb{T}^{k_{1}} \times \mathbb{R}^{k_{2}} \times \mathbb{R}_{+}^{k_{3}}$. In the previous Section we calculate the moment map $\mu_{H}$ associated with the symplectic action of $H$; now using this function we define the following set of functions:

$$
S_{H}=\left\{a: \mu_{H}\left(M_{k}\right) \subset \mathbb{R}^{m} \longrightarrow \mathbb{C}\right\} .
$$

We denote by $\widetilde{S}_{H}=\mu_{H}^{*}\left(S_{H}\right)$ the set of bounded measurable functions $\tilde{a}: M_{k} \rightarrow \mathbb{C}$ such that there exist $a \in S_{H}$ satisfying $\tilde{a}=a \circ \mu_{H}$ and $I_{H}$ the set of bounded functions over $M_{k}$ invariant under the restricted action $\left.\psi\right|_{H}$. Moreover, we know that $\mu_{H}$ is invariant under the action of $H$, so we have that $\widetilde{S}_{H} \subset I_{H}$ for every $H$.

Definition 12. We denote by $\mathscr{T}_{H}$ the $C^{*}$ algebra generated by symbols in $\widetilde{S}_{H}$ where $H$ is an Abelian subgroup of isometries.

In particular, we take a maximal Abelian subgroup $G_{k}$ and consider the injective map $\phi: \Omega_{k} \rightarrow \mathbb{R}^{n}$ defined by

$$
\begin{aligned}
& (r, y, \theta) \longmapsto\left(\frac{1}{2 \pi\left(1-r_{1}^{2}\right)}, \ldots, \frac{1}{2 \pi\left(1-r_{k_{1}}^{2}\right)}, \frac{-1}{4 \pi y_{1}}, \ldots,\right. \\
& \left.\frac{-1}{4 \pi y_{k_{2}}}, \frac{-1}{4 \pi \tan \theta_{1}}, \ldots, \frac{-1}{4 \pi \tan \theta_{k_{3}}}\right),
\end{aligned}
$$

where $z=\left(r t, x+i y, \rho e^{i \theta}\right) \in M_{k}, \phi(r, y, \theta)=\mu_{G_{k}}(z)$, and $\mu_{G_{k}}$ is the moment map associated with $G_{k}$. Thus, we obtain that the image $\phi\left(\Omega_{k}\right) \subset \mathbb{R}^{n}$ coincides with the image $\mu_{G_{k}}\left(M_{k}\right) \subset$ $\mathbb{R}^{n}$. On the other hand, we have that the inverse map $\phi^{-1}$ : $\mu_{G_{k}}\left(M_{k}\right) \rightarrow \Omega_{k}$ is given by

$$
\begin{aligned}
& r_{j}=\sqrt{1-\frac{1}{2 \pi u_{j}}}, \quad j=1, \ldots, k_{1} \\
& y_{j}=-\frac{1}{4 \pi v_{j}}, \quad j=1, \ldots, k_{2} \\
& \theta_{j}=\tan ^{-1}\left(-\frac{1}{4 \pi \xi_{j}}\right), \quad j=1, \ldots, k_{3},
\end{aligned}
$$

with $(u, v, \xi) \in \mu_{G_{k}}\left(M_{k}\right) \subset \mathbb{R}^{n}$. Hence, we obtain that $\phi^{*}\left(I_{G_{k}}\right)=\widetilde{S}_{H}$ and $\left(I_{G_{k}}\right)=\left(\phi^{-1}\right)^{*} \widetilde{S}_{H}$; thus it is clear that $I_{G_{k}}=\widetilde{S}_{H}$.

Therefore, if we consider a maximal Abelian subgroup $G_{k}$, we have that the $C^{*}$-algebra induced by the moment map 
$\mu_{G_{k}}$ is equal to the $C^{*}$-algebra of Toeplitz operators generated by the set of symbols invariant under the action of $G_{k}$. This affirmation is not true for a (nonmaximal) Abelian subgroup of isometries.

Example 13. Let $2 \leq k_{1}=n$ and we consider the Abelian subgroup $H_{0}$ as follows:

$$
t: z \in \mathbb{D}^{n} \longrightarrow\left(t z_{1}, z_{2}, \ldots, z_{n}\right) \in \mathbb{D}^{n}
$$

for each $t \in S^{1}$. A function $a(z), z \in \mathbb{D}^{n}$ is invariant with respect to this action if and only if $a(z)=a\left(r, z_{2}, \ldots, z_{n}\right)$, where $z_{1}=r e^{i \theta}$. It is obvious that Toeplitz operators with this class of symbols do not commute.

On the other hand, we observe that a moment map for $H_{0}$ is given by

$$
\mu_{H_{0}}(z)=\frac{1}{2 \pi\left(1-\left|z_{1}\right|^{2}\right)}
$$

it follows that an element $a \in \widetilde{S}_{H_{0}}$ has the form $a(1 / 2 \pi(1-$ $\left.\left|z_{1}\right|^{2}\right)$ ). A straightforward calculus shows that the $C^{*}$-algebra $\mathscr{T}_{H_{0}}$ is commutative.

Example 14. Let $H_{1}$ be the connected Lie subgroup of $n$-torus $\mathbb{T}^{n}$ which acts as follows:

$$
\begin{aligned}
H_{1} \times \mathbb{D}^{n} & \longrightarrow \mathbb{D}^{n}, \\
(t, \ldots, t) \cdot z & \longmapsto\left(t z_{1}, \ldots, t z_{n}\right) .
\end{aligned}
$$

We denote by $\mathfrak{h}_{1}$ and $\mathfrak{t}^{n}$ the Lie algebras corresponding to $H_{1}$ and $\mathbb{T}^{n}$, respectively. We know that the Lie algebra $\mathfrak{h}_{1}$ is generated by $Y=X_{1}+\cdots+X_{n}$, where $X_{1}, \ldots, X_{n}$ is standard basis of $\mathfrak{t}^{n}$. Hence the moment map associated with $H_{1}$ is given by

$$
\mu_{H_{1}}(z)=\frac{1}{2 \pi} \sum_{i=1}^{n} \frac{1}{1-\left|z_{i}\right|^{2}},
$$

since we have that

$$
i_{Y^{\sharp}} \omega=d \mu_{H_{1}}^{Y} ;
$$

hence Lemma 10 implies that $\mu_{H_{1}}$ is a moment map. We have that every function belonging to $\widetilde{S}_{H_{1}}$ has the form

$$
a(z)=a\left(\frac{1}{2 \pi} \sum_{i=1}^{n} \frac{1}{1-\left|z_{i}\right|^{2}}\right) \text {. }
$$

Thus it is clear that the $C^{*}$-algebra $\mathscr{T}_{H_{1}}$ is commutative.

Remark 15. Consider $H_{0}$ and $H_{1}$ as the above examples; its clear that $H_{0} \cap H_{1}=\{e\}$, where $e$ is the identity in the group. In consequence of the above examples we have that $\mathscr{T}_{H_{0}} \cap \mathscr{T}_{H_{1}}=$ $\{I d\}$, where Id is the operator identity on the Bergman space.

In summary, we have that every Abelian group $H$ of isometries is a subgroup of some maximal Abelian group $G_{k}$. Hence, we have that $\widetilde{S}_{H} \subset \widetilde{S}_{G_{k}}=I_{G_{k}}$. From this we obtain the following theorem.
Theorem 16. If $H$ is a connected Abelian Lie group of biholomorphisms in $\mathbb{D}^{n}$, then the $C^{*}$-algebra $\mathscr{T}_{H}$ is commutative.

Remark 17. Consider $H_{1}$ and $H_{2}$ Abelian Lie groups of biholomorphisms in $\mathbb{D}^{n}$ such that $H_{1}$ is subgroup of $H_{2}$; under the considerations of the above theorem we have that $\mathscr{T}_{H_{1}}$ is subalgebra of $\mathscr{T}_{H_{2}}$. Therefore a chain of commutative Abelian groups of isometries induce a chain of commutative $C^{*}$-algebras.

\section{Competing Interests}

The authors declare that there are no competing interests regarding the publication of this paper.

\section{Acknowledgments}

This work was partially supported by National Council on Science and Technology of México under Grant no. 236109.

\section{References}

[1] S. Grudsky, R. Quiroga-Barranco, and N. Vasilevski, "Commutative $C^{*}$-algebras of Toeplitz operators and quantization on the unit disk," Journal of Functional Analysis, vol. 234, no. 1, pp. 144, 2006.

[2] R. Quiroga-Barranco and N. Vasilevski, "Commutative $C^{*}$ algebras of Toeplitz operators on the unit ball, I. Bargmann-type transforms and spectral representations of Toeplitz operators," Integral Equations and Operator Theory, vol. 59, no. 3, pp. 379419, 2007.

[3] S. Krantz, Function Theory of Several Complex Variables, American Mathematical Society, Providence, RI, USA, 1951.

[4] S. Grudsky, A. Karapetyants, and N. Vasilevski, "Dynamics of properties of Toeplitz operators on the upper half-plane: hyperbolic case," Boletín de la Sociedad Matemática Mexicana, vol. 10, no. 1, pp. 119-138, 2004.

[5] S. Grudsky, A. Karapetyants, and N. Vasilevski, "Dynamics of properties of Toeplitz operators on the upper half-plane: parabolic case," The Journal of Operator Theory, vol. 52, no. 1, pp. 185-204, 2004.

[6] S. Grudsky, A. Karapetyants, and N. Vasilevski, "Dynamics of properties of Toeplitz operators with radial symbols," Integral Equations and Operator Theory, vol. 50, no. 2, pp. 217-253, 2004.

[7] I. Gradshteyn and I. Ryzhik, Tables of Integrals, Series, and Products, Academic Press, Cambridge, Mass, USA, 1980.

[8] R. Quiroga-Barranco and N. Vasilevski, "Commutative $C^{*}$ algebras of Toeplitz operators on the unit ball. II. Geometry of the level sets of symbols," Integral Equations and Operator Theory, vol. 60, no. 1, pp. 89-132, 2008.

[9] F. W. Warner, Foundations of Differentiable Manifolds and Lie Groups, vol. 94 of Graduate Texts in Mathematics, Springer, Berlin, Germany, 1983. 


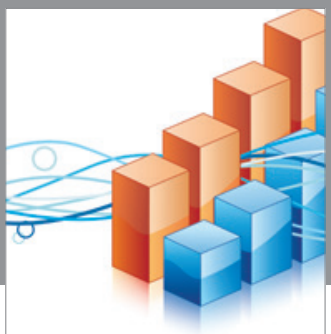

Advances in

Operations Research

vatem alat4

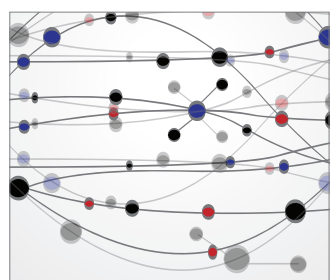

\section{The Scientific} World Journal
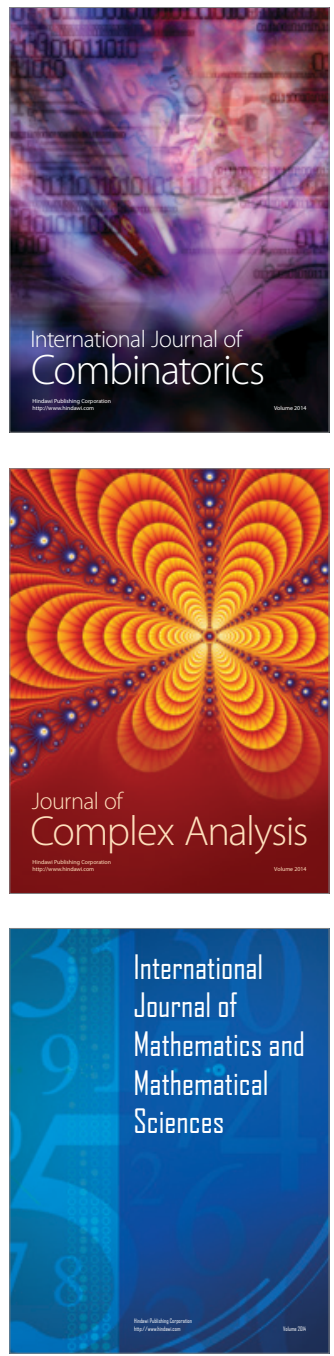
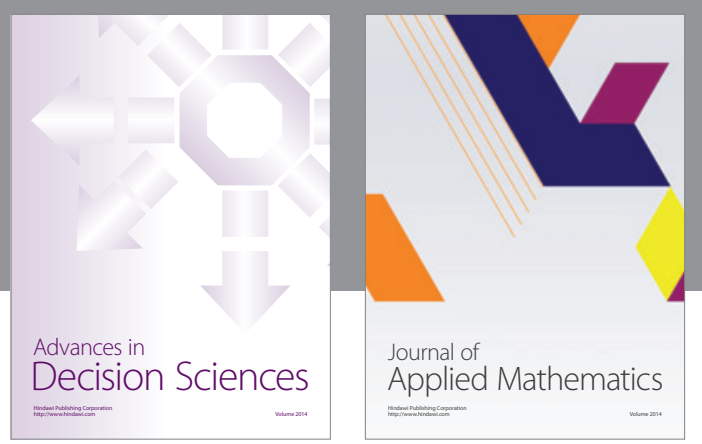

Algebra

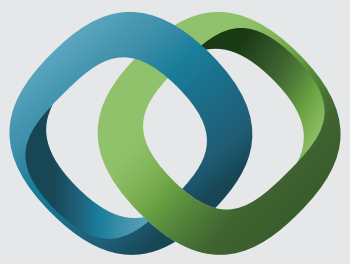

\section{Hindawi}

Submit your manuscripts at

http://www.hindawi.com
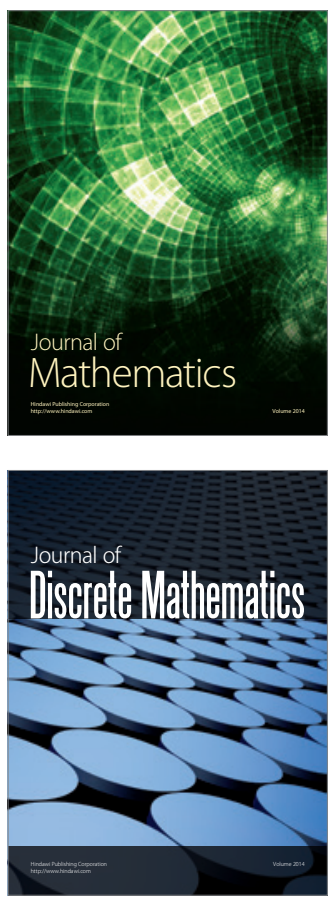

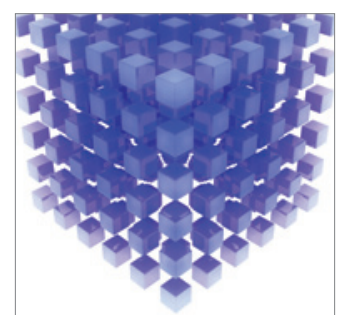

Mathematical Problems in Engineering
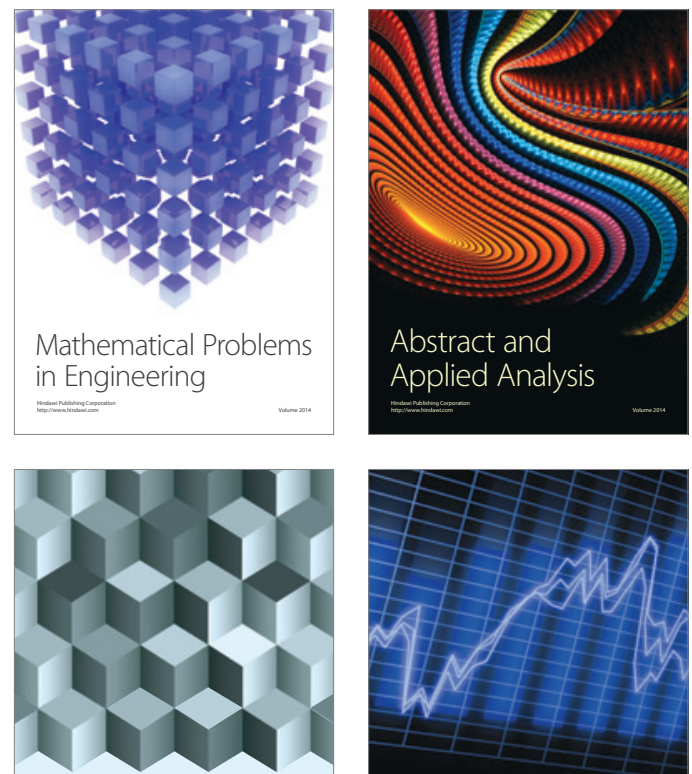

Journal of

Function Spaces

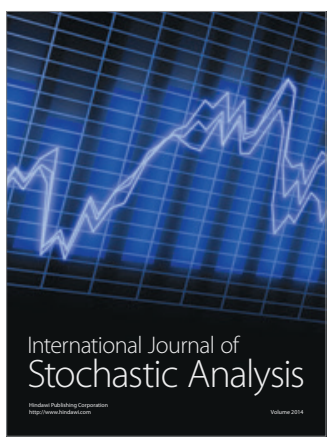

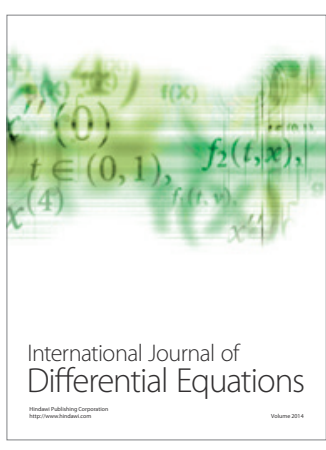
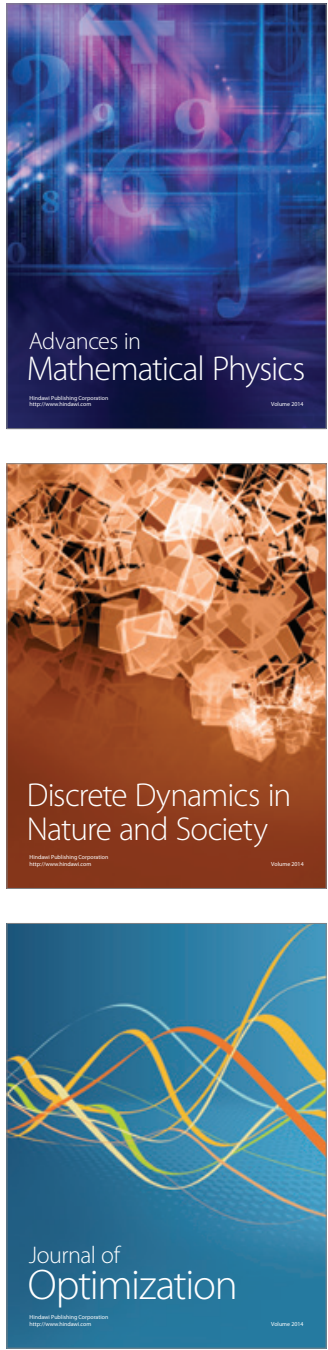